\title{
Robotics in General Surgery: Update and Future Perspectives
}

\author{
Bravo $\mathbf{R}^{\star}$, Arroyave MC, Trépanier JS and Lacy AM
}

Gastrointestinal Surgery, Hospital Clinic Barcelona, Spain

*Corresponding author: Bravo R, Gastrointestinal Surgery, Hospital Clinic Barcelona, Spain, Tel: +34932 275400; E-mail: rbravo.ipaz@gmail.com

Received date: Nov 30, 2015; Accepted date: Dec 19, 2015; Published date: Dec 21, 2015

Copyright: (c) 2015 Bravo R, et al. This is an open-access article distributed under the terms of the Creative Commons Attribution License, which permits unrestricted use, distribution, and reproduction in any medium, provided the original author and source are credited.

\section{Robotics in General Surgery}

Medicine is going through a technological revolution that produces a paradigm shift and makes us think in new ways of treating and diagnosing our patients [1]. Minimally invasive surgery development and routine application in multiple procedures has been the main evolution in the last 50 years, bringing great benefits to patients, surgeons, hospitals and even insurance companies.

In laparoscopic surgery the surgeon keeps control by handling patient's tissues inside an insufflated cavity with an external fulcrum point for instrumentation. It changes drastically in robotic surgery, with the surgeon taking place in a virtual environment outside the operative field, with a distant and indirect control. SAGES defines robotic surgery as a surgical procedure that adds a computer technology enhancement interface to the interaction between the surgeon and his patient during a surgical operation and assumes some degree of control heretofore completely reserved for the surgeon.

Surgical robots have been envisioned to overcome the limitations and extend the capabilities of human surgeons, allowing them to perform precise and reproducible tasks [2]. Its performance is rooted in the strengths and weaknesses of laparoscopic surgery, being able to avoid the fulcrum effect, overcome the limited range of movements and depth perception, and dismiss the surgeon physiological tremor, while keeping its minimally invasive nature [3]

Robotic surgery or computer-assisted surgery is an interactive system fast and intuitive that allows the computer to disappear from the surgeon's mind, who senses as real the environment generated by the system. Through virtual reality, the surgeon defines the manoeuvres that the robot performs in the patient. The consolemanipulator device can be placed in the same operating room, or in a different place, or eventually in another city or country $[4,5]$.

Robotic or remote tele-presence surgery is based in two fundamental concepts: virtual reality and cybernetics. Virtual reality achieves 3D immersion effects, navigation, interaction and simulation in real time, making real what the surgeon sees and touches. Cybernetics makes possible the movement digitalisation, promoting the development of mechanical articulated parts programmed with motion degrees, cameras, sensors, information saving and data processing.

So far tele-presence surgery uses slave robots that are not programmed to do any movement without surgeon's command, and therefore are completely dependent on his judgement, knowledge and skills. It has a structure that resembles the anatomy of human arms and articulations, capable of imitating movements such as those from shoulders, elbows, wrists and fingers, but exceeding its natural range of motion and increasing the degree of freedom. Da Vinci System ${ }^{\infty}$ by Intuitive Surgical Inc, the most commonly used device, consists of a surgeon's console, a slave robot with four interactive arms, instruments, a graphic interface and a camera. This design allows the surgeon to operate from a seated position with ergonomic comfort and enhanced vision of the patient, magnified up to 20 times the real size and in a 3 dimensional fashion. Surgeon's assistant makes the incisions and assembles the arms according to the surgical procedure and the anatomic location of the organ to intervene. Both the optic and instruments movements are originated by the surgeon using no more than two fingers of each hand, and are transmitted to the patient with great precision and dexterity after been interpreted.

Given the constant invasion of new technologies, and adopting a pragmatic point of view, the surgeon has to thoroughly evaluate if robotic surgery adds significant benefits to the procedure in order to translate it into patient well-being. To assess the value of robotic surgery we have to analyse some specific procedures:

Radical prostatectomy is a procedure in which robotics have shown greater advantages over open and laparoscopic surgery, including reduction in postoperative complications, less hospital length of stay and better oncological results [6], but with longer operative times.

Laparoscopic Heller Myotomy is an effective treatment for symptomatic achalasia but with series reporting perforation rates up to $16 \%$. Studies comparing robotic vs laparoscopic myotomy showed perforation rates of 0 vs $6 \%$ respectively, attributing these results to enhanced visualization of muscular layers and more precise movements $[7,8]$.

A meta-analysis [9] reviewing the data of 6 prospective randomized controlled trials including 226 patients compared laparoscopic vs robotic fundoplication. It showed similar results with both techniques but longer operative time and higher cost using robotic surgery.

A systematic review [10] of 9 case series with a total of 130 patients showed better oncologic results in robotic esophagectomy. A higher rate of negative margins and greater number of nodes resected were attributed to a magnified view and easier dissection in a small space as the mediastinum. At Hospital Clinic of Barcelona we are currently performing intrathoracic dissection and anastomosis with Da Vinci xi robot system. We think dissection of upper mediastinum is feasible and safe and could further be benefited by the previously mentioned features of robotic surgery.

A comparative study including 827 patients with gastric cancer compared 236 robotic-assisted to 591 laparoscopic gastrectomies. Mean operative time was 49 minutes longer in robotic surgery, but with significantly less blood loss and better short-term outcomes. Morbidity, mortality and number of nodes resected were comparable in both techniques [11].

For complex procedures such as duodenopancreatectomy, robotic surgery may be useful, as it is a feasible and safe technique [12]. A comparison with open surgery [13] showed less operative time and blood loss with a rise in number of resected nodes. A meta-analysis 
[14] including 6 studies showed an increased rate of R0 resections. The surgeon's opinion was that the robot facilitates the complex steps of the surgery as dissection and reconstruction [15]. Distal pancreatectomy with splenic preservation seems to be another indication for robotassisted surgery, obtaining better oncologic results in preliminary studies [16]

Even though there are only about 100 cases of hepatic robotic resections described in literature, it seems to simplify hepatic pedicle dissection and biliary reconstruction.

A systematic review of colonic resections, including case series and comparative studies showed no benefit of robotic over laparoscopic surgery. For rectal resection there is evidence that robotics reduces conversion to open surgery, with no differences in operative time, morbidity, hospital stay and oncologic results $[17,18]$. Enhanced 3D visualization and more precise manoeuvres of the robot allow nerves preservation during the total mesorectal excision, producing a better recovery of sexual and urinary functions [19]. There are promising advances in transanal proctectomy with completely robotic total mesorrectal excision for rectal cancer with good initial results. It can facilitate transanal total mesorectal excision by providing a tridimensional view, better maneuverability of the robotic instruments and a more comfortable situation for the surgeon, who does not have to work under the technical difficulties and space inherent to TEM or SILS [20].

As attractive as it sounds, there are several limitations to robotic surgery, including the size of the equipment, which limits the space in the operating room and may requires extra staff to operate, higher costs of procedures and unaffordability for some health systems. Lack of haptic (force feedback) and problems with multi-quadrant surgery add additional constraints. Another consideration is that it requires a number of delicate connections and interactions that can be out of control and cause damage to the patient. Besides, the assembling of the device and arms causes a significant rise in operative and anaesthetic times [21].

Many published articles showed no additional benefit of robotic surgery over laparoscopic surgery, with an important increase in the costs [22], bringing to the question of whether or not paying more for the same results is justified. The logical answer is no, but the actual numbers demonstrate a continuous increase in the number of robotic procedures. Despite limitations, some of the results obtained are promising and it seems to be just a matter of time until robotic surgery becomes the gold standard for an important amount of surgical procedures.

We consider the objections that robotic surgery has experienced in the past years are part of a natural path that every new technique has to overcome until it is able to prove that its benefits are greater than the disadvantages. Not long ago, papers like this one were written to compare and evaluate laparoscopic versus open surgery, with very similar considerations. It is no longer a debate that laparoscopic procedures fulfilled the requirements to become the gold standard therapy in multiple fields. Besides the well-proven advantages of less postoperative pain, shorter hospital stays (if necessary at all), a quicker return to daily life and work activities, less risk of infection, reduced pulmonary and thrombotic complications, lower levels of surgical metabolic stress and better cosmetic results [23], also, quality of life is enhanced and morbidity and mortality reduced, achieving the main goals of any health system.
It is now clear that laparoscopic surgery has won against open surgery in many aspects, but it had to conduct many difficult battles to get to the point where it stands today. Initial critiques against laparoscopy included high costs, unknown safety, limited extent of oncological resections and lymphadenectomies, fears of higher trocar wounds and local recurrence in cancer surgery, greater difficulty level of routine surgeries, among others [24]. These concerns were published in multiple journals and medical literature, gaining widespread diffusion and generating a niche for subsequent hundreds if not thousands of investigations trying to address these questions. We are not saying that robotic surgery, as we know it nowadays, is necessarily better than laparoscopic surgery, both being minimally invasive techniques, with advantages and limitations. We want to expose that similar critiques once made toward laparoscopy, when compared to open surgery, are now addressed to robotic surgery.

The robot in its actual form will only evolve and develop into a better system if it gets the opportunity to be used widely. The same history happened with computers, evolving from giant and primitive machines to tiny intelligent devices, showing us that new technologies have to be used repeatedly, in multiple procedures and by different surgeons, in order to get the best out of it. Until procedure-related morbidity and mortality is completely eliminated, the search for better approaches can't be over.

Linda van der Bedem, researcher at Technische Universiteit Eindhoven wrote an article in Science Daily about the development of a more compact surgical robot called Sofie that uses force feedback control [25]. Another research under evaluation is an artificially intelligent surgical robot being developed since 2010 by a group of bioengineers at Duke University. A robot that will be able to find a lesion in simulated tissues and guide a device towards the lesion to take samples or biopsies (Duke robot Biopsy guided by 3-D ultrasound).

Advances have been made in the development and implementation of single-port robotic surgery, showing feasibility and efficacy in cholecystectomy and mayor urologic procedures [26,27].

This race is not over as development of nano-robots takes the lead. These are robots the size of a cell that can be introduced in blood flow to eliminate cancer cells, tissue repair or toxic radicals capture, among other uses.

As responsible health providers, we have the obligation to review our results transparently and precisely, and take directions based on these evaluations in order to avoid overcosts, damage to patients or the use of therapies with futile benefits, but there has to be a balance between thoughtful assessment and innovative research, so new technologies have the time and opportunity to show their merits and establish themselves. Only history will tell us if it is only market pressure and the manufacturer's drive what keeps the robot standing. We would like to think that consolidation of techniques, achievement of staff learning curves and lower prices as market expands will produce the cost effective results we expect, allowing it to stand up for itself.

Although future is uncertain in many aspects of life, it seems to be full of exciting possibilities for robotic surgery. Everyday we witness new developments that bring surgery closer to the digital age showing us that future of robotics is limited only by imagination. 


\section{References}

1. Portillo MM, Salazar VC, Guadarrama QCD, Gahbler PC, Rodrıguez (RM 2014) Cirugía robótica. Gac Med Mex 3: 293-7.

2. Herron DM, Marohn M (2008) A consensus document on robotic surgery. Surg Endosc 22: 213-25.

3. Stefanidis D, Wang F, Korndorffer JJ, Dunne JB, Scott DJ (2010) Robotic assistance improves intracorporeal suturing performance and safety in the operating room while decreasing operator workload. Surg Endosc 24: 377-82.

4. Marescaux J, Leroy J, Rubino F, Smith M, Vix M, et al. (2002) Transcontinental robot-assisted remote telesurgery: Feasibility and potential applications. Ann Surg 235: 487-92.

5. Lanfranco AR, Castellanos AE, Desai JP, Meyers WC (2004) Robotic surgery: A current perspective. Ann Surg 239: 14-21.

6. Alemozaffar M, Sanda M, Yecies D, Mucci LA, Stampfer MJ, et al. (2015) Benchmarks for operative outcomes of robotic and open radial prostatectomy: Results from the Health Professionals Follow-up Study. Eur Urol 67: 432-8.

7. Horgan S, Galvani C, Gorodner MV, Omelanczuck P, Elli F, et al. (2005) Robotic-assisted Heller myotomy versus laparoscopic Heller myotomy for the treatment of esophageal achalasia: Multicenter study. J Gastrointest Surg 9: 1020-9.

8. Melvin WS, Dundon JM, Talamini M, Horgan S (2005) Computerenhanced robotic telesurgery minimizes esophageal perforation during Heller myotomy. Surgery 138: 553-8.

9. Markar SR, Karthikesalingam AP, Hagen ME, Talamini M, Horgan S, et al. (2010) Robotic vs. laparoscopic Nissen fundoplication for gastrooesophageal reflux disease: Sytematic review and meta-analysis. Int J Med Robot 6: 125-31.

10. Clark J, Sodergren MH, Purkayastha S, Mayer EK, James D, et al. (2011) The role of robotic assisted laparoscopy for oesophagogastric oncological resection; an appraisal of the literature. Dis Esophagus 24: 240-50.

11. Woo Y, Hyung WJ, Pak KH, Inaba K, Obama K, et al. (2011) Robotic gastrectomy as an oncologically sound alternative to laparoscopic resections for the treatment of early-stage gastric cancers. Arch Surg 146: 1086-92.

12. Giulianotti PC, Sbrana F, Bianco FM, Elli EF, Shah G, et al. (2010) Robotassisted laparoscopic pancreatic surgery: Single-surgeon experience. Surg Endosc 24: 1646-57.

13. Buchs NC, Addeo P, Bianco FM, Ayloo S, Benedetti E, et al. (2011) Robotic versus open pancreaticoduodenectmy: A comparative study at a single institution. World J Surg 35: 2739-46.
14. Chen Y, Yan J, Yuan Z, Yu S, Wang Z, et al. (2013) A meta-analysis of robotic-assisted pancreatectomy versus laparoscopic and open pancreatectomy. Saudi Med J 34: 1229-36.

15. Nguyen KT, Zureikat AH, Chalikonda S, Bartlett DL, Moser AJ, et al. (2011) Technical aspects of robotic-assisted pancreaticoduodenectomy (RAPD). J Gastrointest Surg 15: 870-5.

16. Daouadi M, Zureikat AH, Zenati MS, Choudry H, Tsung A, et al. (2013) Robot-assisted minimally invasive distal pancreatectomy is superior to the laparoscopic technique. Ann Surg 257: 128-32.

17. Memon S, Heriot AG, Murphy DG, Bressel M, Lynch AC (2012) Robotic versus laparoscopic proctectomy for rectal cancer: A meta-analysis. Ann Surg Oncol 19: 2095-101.

18. Trastulli S, Farinella E, Cirocchi R, Cavaliere D, Avenia N, et al. (2012) Robotic resection compared with laparoscopic rectal resection for cancer: Systematic review and meta-analysis of short-term outcome. Colorectal Dis 14: 134-56.

19. Kim JY, Kim NK, Lee KY, Hur H, Min BS (2012) A comparative study of voiding and sexual function after total mesorectal excision with autonomic nerve preservation for rectal cancer: Laparoscopic versus robotic surgery. Ann Surg Oncol 19: 2485-93.

20. Ruiz GM, Parra IM, Palazuelos CM, Martín JA, Fernández CC, et al. (2015) Robotic-Assisted laparoscopic transanal total mesorectal excision for rectal cancer. Dis Col Rect 58: 146-146.

21. Sung GT, Gill IS (2001) Robotic laparoscopic surgery: A comparison of the da Vinci and ZEUS systems. Urology 58: 893-8.

22. Barbash G, Glied S (2010) New Technology and Health Care Costs-The Case of Robot-Assisted Surgery. The New England Journal of Medicine 363: 701-704.

23. Wilson EB (2009) The evolution of robotic general surgery. Scand J Surg 98: 125-9.

24. Serra SA (2012) Tecnologia o tecnolatría: Hacia dónde van los cirujanos? Cir Esp 90: 156-61.

25. Bedem VL (2010) Better surgery with new surgical robot with force feedback. Eindhoven University of Technology.

26. Wren SM, Curet MJ (2011) Single-Port Robotic Cholecystectomy: Results From a First Human Use Clinical Study of the New da Vinci Single-Site Surgical Platform. Arch Surg 146: 1122-1127.

27. Kaouk JH, Haber GP, Autorino R, Crouzet S, Ouzzane A, et al. (2014) A novel robotic system for single-port urologic surgery: First clinical investigation. Eur Urol 66: 1033-1043. 